\title{
Meridional almost normal surfaces in knot complements
}

\author{
ROBIN TODD WILSON
}

\begin{abstract}
Suppose $K$ is a knot in a closed 3-manifold $M$ such that $M-N(K)$ is irreducible. We show that for any integer $n$ there exists a triangulation of $M-N(K)$ such that any weakly incompressible bridge surface for $K$ of $n$ bridges or fewer is isotopic to an almost normal bridge surface.
\end{abstract}

$57 \mathrm{M} 99$

\section{Introduction}

It was shown independently by M Stocking [14] and J H Rubinstein [11] that any strongly irreducible Heegaard splitting for an irreducible 3-manifold is isotopic to an almost normal surface. Also see S King [10] for another proof of this result. The concept of a bridge surface for a knot complement is analogous to the idea of a Heegaard surface for a 3-manifold in that the bridge surface is a splitting surface that separates the knot complement into two equivalent and fairly elementary submanifolds. In addition, the fact that a bridge surface lifts to a Heegaard surface in the 2-fold branched cover of a knot complement gives another important connection between bridge surfaces for knot complements and Heegaard surfaces for 3-manifolds.

In the study of bridge surfaces for knots and links the idea of a weakly incompressible splitting surface is immediately analogous to the idea of a strongly irreducible Heegaard surface for a 3-manifold. In this paper we prove an analog to the main theorem of Stocking [14] and Rubinstein [11, Theorem 3]. We show that any weakly incompressible bridge surface in a 3-manifold is isotopic to an almost normal bridge surface.

Main Theorem 1 Let $K$ be a knot in a closed, orientable, irreducible 3-manifold $M$. Let $N(K)$ be an open regular neighborhood of $K$ and suppose that the complement $M_{K}=M-N(K)$ is irreducible. Then for any integer $n$ there is a triangulation $\mathcal{T}$ of $M_{K}$ such that if $S$ is a bridge surface for $K$ of $n$ bridges or fewer that gives an irreducible Heegaard splitting of $M$ and $S_{K}=S-N(K)$ is weakly incompressible, then $S_{K}$ is properly isotopic in $M_{K}$ to an almost normal surface with respect to $\mathcal{T}$. 
The proof will be similar in spirit to that of [14] but the proof here fills in a few missing cases and simplifies the argument by making greater use of edge slides. Closely related results have been proven by David Bachman [1] and Alexander Coward [4]. In Section 2 we briefly introduce some definitions and notation. The proof of the main theorem is contained in Section 3.

This research was done while under the support of the UC President's Postdoctoral Fellowship Program and the Department of Mathematics at UC Santa Barbara. I would like to thank Martin Scharlemann for all of the helpful conversations and many valuable comments, as well as Scott Taylor for his insightful comments about the proof of Lemma 24.

\subsection{Acknowledgments}

Research supported by UC President's Postdoctoral Fellowship Program and Department of Mathematics at the University of California, Santa Barbara.

\section{Preliminaries}

Notation If $K$ is a properly embedded 1-manifold in a 3-manifold $M$ then let $M_{K}=M-N(K)$. If $X$ is any surface in $M$ transverse to $K$ such that $K \cap X \neq \varnothing$, then let $X_{K}=M_{K} \cap X$. For $\mathcal{T}$ a triangulation of a 3-manifold $M$, let $\mathcal{T}_{\partial M}$ denote the restriction of $\mathcal{T}$ to $\partial M$.

The following definition is from Tomova [16] and is based on the definition of a $K$-compression body given in Bachman [2].

Definition 2 ([16]) A properly embedded arc $K$ in a 3-manifold $M$ is boundary parallel if there is a disk $D$ in the 3-manifold so that $\partial D$ is the end point union of $K$ and an arc in $\partial M$. The disk $D$ is called a cancelling disk for $K$. A $K$-handlebody $(A, K)$ is a handlebody $A$ containing a finite collection of boundary parallel $\operatorname{arcs} K$. When there is little risk of confusion we will also refer to $A_{K}=A-N(K)$ as a $K$-handlebody. For our purposes, a $K$-compression body $(W, K)$ is a compression body $W$ containing a finite collection of arcs $K$ properly embedded in $W$ such that each arc is either boundary parallel or each arc has one end on each of $\partial_{+} W$ and $\partial_{-} W$ and is vertical in the product region $\partial_{-} W \times I \subset W$.

Remark 3 Two sets $K$ and $K^{\prime}$ of boundary parallel arcs in a handlebody $A$ or vertical arcs in a compression body are properly isotopic in $A$ if they have the same cardinality, ie, $|K|=\left|K^{\prime}\right|$. 
Definition 4 A spine of a handlebody $A$ is a graph $\Sigma_{A}$ properly embedded in $A$ such that $A-\Sigma_{A}$ is a product $\partial A \times I$. Let $(A, K)$ be a $K$-handlebody and suppose that $\Sigma_{A}$ is a spine for handlebody $A$ and $K$ is a collection of boundary parallel arcs in $A$. Let $\alpha$ be a collection of $|K|$ arcs, each connecting $\Sigma_{A}$ to a single arc of $K$. Then a regular neighborhood $A^{\prime}=N\left(\Sigma_{A} \cup \alpha\right)$ is again a $K$-handlebody, and $K$ intersects it in a boundary parallel set of arcs $K^{\prime} \subset A^{\prime}$. If the closure of the region $\left(A-A^{\prime}\right)_{\left(K-K^{\prime}\right)}$ between them is a product $\partial A_{K} \times I$ then $\Sigma_{(A, K)}=\Sigma_{A} \cup \alpha$ is called a spine of the $K$-handlebody $(A, K)$.

Definition 5 A spine of a compression body $W$ is a graph $\Sigma_{W}$ with endpoints in $\partial_{-} W$ such that $W-\left(\Sigma_{W} \cup \partial_{-} W\right)$ is a product $\partial_{+} W \times I$. Let $(W, K)$ be a $K$-compression body and suppose that $\Sigma_{W}$ is a spine for $W$ and $K$ is a collection of boundary parallel and vertical arcs in $W$. Let $k$ denote the collection of boundary parallel arcs in $K$. Let $\beta$ be a collection of $|k|$ arcs, each connecting $\Sigma_{W}$ to a single arc of $k$. Then a regular neighborhood $W^{\prime}=N\left(\Sigma_{W} \cup \beta \cup \partial_{-} W_{K}\right)$ is again a $K$-compression body and $K$ intersects it in a set of boundary parallel and vertical arcs $K^{\prime} \subset W^{\prime}$. If the closure of the region $\left(W-W^{\prime}\right)_{\left(K-K^{\prime}\right)}$ between them is a product $\partial_{+} W_{K} \times I$ then $\Sigma_{(W, K)}=\Sigma_{W} \cup \beta$ is called a spine of the $K$-compression body $(W, K)$.

Remark 6 It is relatively easy to find such a spine for a $K$-handlebody or $K-$ compression body $(A, K)$. Choose a spine for $\Sigma$ of handlebody (compression body) $A$ and isotope $K$ so that in the product structure $A-N(\Sigma)=\partial A \times I$, each (boundary parallel) arc of $K$ has a single maximum. Let $\alpha(\beta)$ be a collection of vertical arcs in this product structure, connecting each maximum of $K$ to $\Sigma$.

Definition 7 (Scharlemann-Tomova [13]) Let $K$ be a knot in a closed, orientable 3-manifold $M$ and let $S$ be a Heegaard surface for $M$. That is, $M=W \cup_{S} W^{\prime}$, where $W$ and $W^{\prime}$ are handlebodies in $M$. If in addition, $W_{K}$ and $W_{K}^{\prime}$ are $K-$ handlebodies then we call $S$ a bridge surface for $M_{K}$. (We will often abuse notation and call the punctured surface $S_{K}$ a bridge surface as well.) We call the decomposition $M_{K}=W_{K} \cup_{S_{K}} W_{K}^{\prime}$ a bridge splitting of the 3-manifold $M_{K}$ and we say that $K$ is in bridge position with respect to bridge surface $S$.

Definition 8 ([13]) Let $K$ be a $1-$ manifold embedded in $M$ and suppose that $F$ is a properly embedded surface in $M$ so that $F$ is transverse to $K$. A simple closed curve on $F_{K}$ is essential if it doesn't bound a disk or a once punctured disk in $F_{K}$. An embedded disk $D \subset M_{K}$ is a compressing disk for a surface $F_{K}$ if $D \cap F_{K}=\partial D$ and $\partial D$ is an essential curve in $F$. A surface $F$ in $M$ is a splitting surface for $M$ if we can express $M$ as the union of two 3-manifolds along $F$. If $F$ is a splitting 
surface for $M$ then we say that the surface $F_{K}$ is weakly incompressible if any pair of compressing disks on opposite sides of the surface intersect. If $F_{K}$ compresses on both sides but is not weakly incompressible then it is called strongly compressible.

The study of normal surfaces was first developed by Haken [7]. The concept of an almost normal surface that is used in this paper first appeared in [11].

Definition 9 Let $S$ be a triangulated surface and let $c$ be a curve on $S$. Assume that $c$ is transverse to the 1-skeleton of the triangulation. A curve $c$ in $S$ is called normal if the intersection of $c$ with any triangle of the triangulation contains no closed curves and no arcs with both endpoints on the same edge.

Definition 10 ([7]) Let $M$ be a triangulated 3-manifold. A normal triangle in a tetrahedron of the triangulation is an embedded disk that meets three edges and three faces of the tetrahedron. A normal quadrilateral is an embedded disk in a tetrahedron that meets four edges and four faces of the tetrahedron. Normal triangles and quadrilaterals are called normal disks. Normal disks meet the faces of the boundary of a tetrahedron in normal curves.

Definition 11 ([11]) Let $M$ be a triangulated 3-manifold. An embedded surface $S \subset M$ is a normal surface if it meets each tetrahedron in a disjoint collection of normal disks. A surface $S$ is almost normal if $S$ meets each tetrahedron of the triangulation in a collection of normal disks, but in one tetrahedron there is exactly one exceptional piece. This exceptional piece is either a normal octagon, or it is an annulus consisting of two normal disks with a tube between them that is parallel to an edge of the 1-skeleton.

The proof of the main theorem relies heavily on the idea of thin position, first introduced by Gabai [6].

Definition 12 ([14]) Let $M_{K}=W_{K} \cup_{S_{K}} W_{K}^{\prime}$ denote a bridge splitting of $M_{K}$. Given spines $\Sigma_{(W, K)}$ and $\Sigma_{\left(W^{\prime}, K\right)}$ for the $K$-handlebodies $(W, K)$ and $\left(W^{\prime}, K\right)$ respectively, there is a diffeomorphism $S_{K} \times(0,1) \simeq M_{K}-N\left(\Sigma_{(W, K)} \cup \Sigma_{\left(W^{\prime}, K\right)}\right)$. For $t \in(0,1)$ denote the surface corresponding to $S_{K} \times\{t\}$ by $S_{t} \subset M_{K}$. A standard singular foliation $F$ of $M_{K}=W_{K} \cup_{S_{K}} W_{K}^{\prime}$ extends this structure to all of $M_{K}$ by adding two singular leaves $S_{0}$ and $S_{1}$, called the top and bottom leaves. All leaves meet the torus $\partial M_{K}$ in the standard foliation in meridian circles. The top and bottom singular leaves consist of the union of the spines of the $K$-handlebodies $W_{K}$ and $W_{K}^{\prime}$ respectively and the meridian circles of $\partial M_{K}$ corresponding to each of the $n$ endpoints of $\Sigma_{(W, K)}$ and $\Sigma_{\left(W^{\prime}, K\right)}$. There is a height function $h: M \rightarrow[0,1]$ associated with the standard singular foliation given by the map that sends all points on a leaf $S_{t}$ together with the incident meridian disks of $N(K)$ to the point $t$ in $[0,1]$. 
Definition 13 (Thompson [15] and Stocking [14]) Assume that $T$ is a collection of arcs properly embedded in $M_{K}$ and is in general position with respect to $F$, a standard singular foliation of $M_{K}$. That is, all but a finite number of leaves of $F$ intersect $T$ transversally, every leaf in $F$ has at most one point of tangency with $T$ and $T$ is disjoint from the singular subarcs of the singular leaf. If a leaf has a point of tangency with $T$ call it a tangent leaf and all other leaves transverse leaves. Between each two adjacent tangent leaves choose a transverse leaf $L_{i}$. Define the width of a fixed embedding of $T$ with respect to $F$ to be the sum over $i$ of the number of times $T$ intersects $L_{i}$. If $T$ has been properly isotoped to minimize its width with respect to $F$ then we say that $T$ is in thin position with respect to $F$.

Definition $14([15 ; 14])$ Let $T$ be in thin position with respect to a standard singular foliation $F$. Then as we move down the foliation from the top arcs will form a sequence of maxima with respect to $F$, then a sequence of minima and so on. We will call a leaf in a region where the sequence shifts from maxima to minima a thick leaf and we will call such a region a thick region. An upper (lower) disk $D$ for a transverse leaf $L$ of $F$ is a disk in $\operatorname{int}(M)-T$ such that $\partial D=\alpha \cup \beta$ where $\alpha$ is an arc embedded in $L, \beta$ is a subarc of $T, \partial \alpha=\partial \beta, D-\alpha$ intersects $L$ transversely and a small neighborhood of $\alpha$ lies above (below) $L$.

For the proof of the main theorem we will need the following Lemmas. The first Lemma is proved by Stocking in [14].

Lemma 15 ([14, Lemma 1]) Let $S$ be an almost normal surface in an irreducible 3manifold. Suppose that $S$ is incompressible to one side. Then $S$ is isotopic to a normal surface that does not intersect $S$ and that does not contain $S$ to the incompressible side.

A version of the following theorem was proved for strongly irreducible Heegaard surfaces by Casson and Gordon in [3] and has been adapted to the situation of weakly incompressible bridge surfaces by Tomova in [16].

Lemma 16 ([16, Corollary 6.3]) Let $K$ be a knot in a closed, orientable, irreducible 3-manifold $M$. Let $S_{K}$ be a weakly incompressible splitting surface for $M_{K}$ and let $S_{K}^{\prime}$ be a surface that is obtained from $S_{K}$ by compressing $S_{K}$ to one side. Then $S_{K}^{\prime}$ is incompressible to the other side. 


\section{Almost normal bridge surfaces}

The proof of the main theorem follows from an application of ideas from [14] where the Heegaard surface is replaced with a bridge surface. An important difference between the two arguments is that the leaves of the foliations in this case are surfaces with boundary as opposed to closed surfaces. The argument relies heavily on Lemma 17, Lemma 24 and Lemma 25 whose statements are close to those of [14, Lemmas 4 and 5]. The proofs of these lemmas are similar in spirit to the originals, but differ in detail. Several cases missed in the original proof in [14] are included here, more extensive use is made of edgeslides and the arguments have been adapted to our situation.

Proof of Main Theorem Let $K$ be a knot in a closed, orientable 3-manifold $M$ and assume that $M$ and $M_{K}$ are both irreducible. Suppose that the knot $K$ is in $n$-bridge position with bridge surface $S$ so that $M=W \cup_{S} W^{\prime}$ is an irreducible Heegaard splitting of $M$ and the punctured surface $S_{K}$ is weakly incompressible. Also assume that $S_{K}$ separates $M_{K}$ into the two $K$-handlebodies $W_{K}$ and $W_{K}^{\prime}$. Let $M_{K}=W_{K} \cup_{S_{K}} W_{K}^{\prime}$ denote the bridge splitting of $M$ by $S$. We can foliate $M_{K}=W_{K} \cup_{S_{K}} W_{K}^{\prime}$ with a standard singular foliation that intersects the torus $\partial M_{K}$ in meridian circles. The top singular leaf of the foliation, $L_{\text {top }}$, is a 1-complex given by the union of a spine $\Sigma_{(W, K)}$ of $W_{K}$ and one meridian circle of $\partial M_{K}$ for each of the $n$ endpoints of $\Sigma_{(W, K)}$ on $K$. Similarly, the bottom singular leaf of the foliation, $L_{\text {bot }}$, is a 1-complex given by the union of a spine $\Sigma_{\left(W^{\prime}, K\right)}$ of $W_{K}^{\prime}$ and one meridian circle of $\partial M_{K}$ for each of the $n$ endpoints of $\Sigma_{\left(W^{\prime}, K\right)}$ on $K$. Thus there is a symmetric picture near the top and bottom leaves of the foliation.

Consider a nearby leaf of the frontier of a regular neighborhood of $L_{\text {top }}$ (resp. $L_{\text {bot }}$ ) in $M_{K}$. It can be viewed as consisting of two parts. See Figure 1. The first is a collection $\Gamma^{\text {top }}$ (resp. $\Gamma^{\text {bot }}$ ) of $n$ boundary parallel annuli. Secondly, these annuli are tubed together via the boundary $t^{\text {top }}$ (resp. $t^{\text {bot }}$ ) of a regular neighborhood of $\Sigma_{(W, K)}$ (resp. $\left.\Sigma_{\left(W^{\prime}, K\right)}\right)$. Topologically $\Gamma^{\text {top }}$ (resp. $\Gamma^{\text {bot }}$ ) consists of $n$ once-punctured annuli and $t^{\text {top }}\left(\right.$ resp. $t^{\text {bot }}$ ) consists of an $n$-punctured copy of $S_{K}$. Since $t^{\text {top }}$ and $t^{\text {bot }}$ arise as the boundary of a regular neighborhood of a 1-complex it is natural to refer to them as collections of "tubes". We will refer to the collection of annuli $\Gamma^{\text {top }}$ and $\Gamma^{\text {bot }}$ as $\Gamma$. Throughout the paper the foliation we refer to will always be the standard product foliation of $S^{1} \times I$ on each component between the top and bottom annuli on $\partial M_{K}$.

Next we will describe how to triangulate $M_{K}$ so that the collection $\Gamma$ of annuli is normal with respect to the triangulation. See Figure 2. Consider the collection of $2 n$ meridional annuli on $\partial M_{K}$ parallel to $\Gamma$. Start by choosing a longitude of $\partial M_{K}$. Next, in each annulus choose a meridian circle. View the intersection of each meridian and 


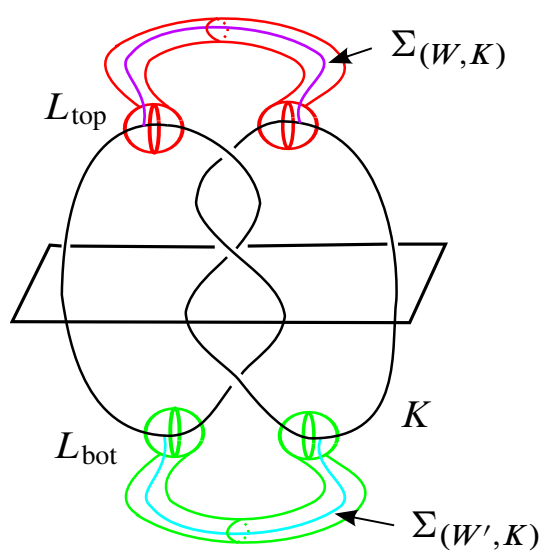

Figure 1: An example with $M=S^{3}$ and $K$ a trefoil

the longitude as a vertex, view each of these meridians as the union of a vertex and an edge and view the longitude as a union of $2 n$ edges and $2 n$ vertices. This divides $\partial M_{K}$ into rectangles. Now subdivide each rectangle by adding a diagonal edge connecting two adjacent vertices. This gives a triangulation of $\partial M_{K}$.

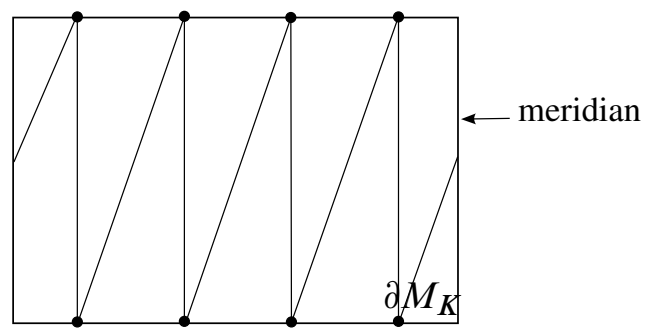

Figure 2: Triangulation of $\partial M_{K}$

By Jaco-Letscher-Rubinstein [8] (also see Jaco-Rubinstein [9, page 56]) we can extend the triangulation of $\partial M_{K}$ to a triangulation of all of $M_{K}$ without adding any vertices. Denote this triangulation of $M_{K}$ by $\mathcal{T}$. All of the vertices of this triangulation are contained in a neighborhood of the top and bottom leaves of the foliation. The collection $\Gamma$ of $2 n$ annuli is normal with respect to this triangulation and $\Gamma$ contains all of the $2 n$ vertices of the triangulation to one side, separating them from the rest of $M_{K}$. Also note that $\mathcal{T}$ has all of its vertices on $\partial M_{K}$, so it has no vertex-linking 2 -spheres. However, $\mathcal{T}$ may contain normal 2 -spheres disjoint from $\Gamma$ that are not vertex-linking. 
Let $\Lambda$ denote a maximal collection of non-parallel disjoint normal 2-spheres in $M_{K}$ disjoint from $\Gamma$. Cutting $M_{K}$ open along $\Lambda$ results in several components, but only one component will contain the torus boundary of $M_{K}$. Call this component $M_{0}{ }^{+}$. Note that $M_{0}{ }^{+}$may have multiple 2-sphere boundary components along with $\partial M_{K}$. Since $M_{K}$ is irreducible each 2-sphere in $\Lambda$ must bound a 3-ball to the opposite side of $M_{0}^{+}$.

Since the annuli $\Gamma$ are normal and they contain the vertices of the triangulation to one side, each normal 2-sphere in $\partial M_{0}{ }^{+}$is connected to $\Gamma$ via an edge of $\mathcal{T}^{1}$. If an edge of $\mathcal{T}^{1}$ connects two 2 -sphere boundary components of $M_{0}{ }^{+}$then by Thompson [15, Lemma 2] it follows that $M_{0}{ }^{+}$must be a punctured 3-ball with a torus boundary component, which it clearly is not. Thus we can conclude each 2-sphere component of $\partial M_{0}{ }^{+}$is connected to $\Gamma^{\text {top }}$ or $\Gamma^{\text {bot }}$ by an edge of $\mathcal{T}^{1} \cap M_{0}{ }^{+}$. Assume without loss of generality that an edge connects the $2-$ sphere to $\Gamma^{\text {top }}$. Taking a tube that lies in the interior of a tetrahedron parallel to this edge connecting $\Gamma^{\text {top }}$ to the normal 2-sphere gives an almost normal annulus isotopic to an annulus in $\Gamma^{\text {top }}$. By Lemma 15 this surface is isotopic to a normal surface giving a new collection of normal annuli that we will call $\Gamma^{\mathrm{top}^{\prime}}$. We can isotope the tubes $t$ so that they lie in $M_{0}{ }^{+}$. We can do this for each normal 2-sphere in $M_{0}{ }^{+}$and then replace the original singular leaf $L_{\text {top }}$ with the singular leaf $L_{\text {top }}^{\prime}=\Gamma^{\mathrm{top}^{\prime}} \cup \Sigma_{(W, K)}$. Let $M_{0}$ be the side of $\Gamma^{\mathrm{top}^{\prime}}$ that lies in $M_{0}{ }^{+}$.

Let $K_{0}=K \cap M_{0}$. Isotoping the bridge surface $S_{K}$ to be disjoint from $\Gamma^{\prime}$ induces a splitting of $M_{0}$ into two $K_{0}$-compression bodies $W_{0}$ and $W_{0}^{\prime}$. Continue to call this splitting surface $S_{K}$. Since $M_{0}$ is a deformation retract of $M_{K}$ the surface $S_{K}$ is a weakly incompressible splitting surface for $M_{0}$. We can foliate $M_{0}$ with a standard singular foliation $F_{0}$ with leaves isotopic to $S_{K}$. The top leaf of the foliation is $L_{\text {top }}$ and the bottom leaf is $L_{\text {bot }}$. Let $\mathcal{T}_{0}^{1}$ denote the part of the 1 -skeleton of $\mathcal{T}$ that lies in the interior of $M_{0}$. Put $\mathcal{T}_{0}^{1}$ into thin position with respect to $F_{0}$. Let $\Sigma_{0}$ denote the pair of spines $\Sigma_{\left(W_{0}, K_{0}\right)}$ and $\Sigma_{\left(W_{0}^{\prime}, K_{0}\right)}$ of the $K_{0}$-compression bodies $W_{0}$ and $W_{0}^{\prime}$ respectively. Note that $\Sigma_{\left(W_{0}, K_{0}\right)} \subset \Sigma_{(W, K)}$ and $\Sigma_{\left(W_{0}^{\prime}, K_{0}\right)} \subset \Sigma_{\left(W^{\prime}, K\right)}$. If $\mathcal{T}_{0}^{1}$ intersects $\Sigma_{0}$ then isotope $\mathcal{T}_{0}^{1}$ slightly off of $\Sigma_{0}$. Let $\Gamma_{0}$ denote the pair $\Gamma^{\text {top }}{ }^{\prime}$ and $\Gamma^{\text {bot' }^{\prime}}$.

The triple $\left(M_{0}, \Sigma_{0}, \Gamma_{0}\right)$ is the first step in an iterative process. Each later step will consist of a triple $\left(M_{i}, \Sigma_{i}, \Gamma_{i}\right)$ with the following properties: $M_{i} \subset M_{i-1}$ will be a submanifold of $M_{K}$ for each $i \geq 0$. The surface $\Gamma_{i}=\partial M_{i}-\partial M$ will be a pair $\Gamma_{i}^{\text {top }}$ and $\Gamma_{i}^{\text {bot }}$ of properly embedded normal surfaces with respect to the triangulation $\mathcal{T}$ given above. Let $K_{i}=K \cap M_{i}$. The submanifold $M_{i}$ has a weakly incompressible splitting surface $S_{K}$ that separates $M_{i}$ into two $K_{i}$-compression bodies $W_{i}$ and $W_{i}^{\prime}$. Let $\Sigma_{i}^{\text {top }}$ and $\Sigma_{i}^{\text {bot }}$ denote the spines $\Sigma_{\left(W_{i}, K_{i}\right)}$ and $\Sigma_{\left(W_{i}^{\prime}, K_{i}\right)}$ of $K_{i}$-compression 
bodies $W_{i}$ and $W_{i}^{\prime}$ respectively. The spine $\Sigma_{i}^{\text {top }}$ (resp. $\Sigma_{i}^{\text {bot }}$ ) can be extended to give a spine of $M-\Sigma_{W^{\prime}} \simeq W$ (resp. $M-\Sigma_{W} \simeq W^{\prime}$ ). Let $\Sigma_{i}$ denote the pair of spines $\Sigma_{i}^{\text {top }}$ and $\Sigma_{i}^{\text {bot }}$ of $W_{i}$ and $W_{i}^{\prime}$ respectively. As usual we define $\Sigma_{i}$ up to isotopy and slides of edges over other edges and over $\partial_{-} W_{i}=\Gamma_{i}^{\text {top }}$. The complement of a regular neighborhood $N\left(\Sigma_{i}^{\text {top }}\right)$ (resp. $N\left(\Sigma_{i}^{\text {bot }}\right)$ ) in $M_{i}$ is foliated by copies of $S_{K}$ with singular leaf $\Gamma_{i}^{\text {bot }} \cup \Sigma_{i}^{\text {bot }}$ (resp. $\Gamma_{i}^{\text {top }} \cup \Sigma_{i}^{\text {top }}$ ). Thus $M_{i}$ can be foliated with a singular foliation $F_{i}$ by copies of $S_{K}$ with its top singular leaf consisting of $\Gamma_{i}^{\text {top }} \cup \Sigma_{i}^{\text {top }}$ and its bottom singular leaf consisting of $\Gamma_{i}^{\text {bot }} \cup \Sigma_{i}^{\text {bot }}$. Now consider the edges of $\mathcal{T}^{1}$ that do not lie on $\partial M_{K}$ and let $\mathcal{T}_{i}^{1}$ denote their intersection with $M_{i}$. Put $\mathcal{T}_{i}^{1}$ into thin position with respect to $F_{i}$.

In general we will obtain the submanifold $M_{i}$ from $M_{i-1}$ for each $i \geq 1$ by showing that $M_{i-1}$ contains an almost normal surface obtained by compressing the splitting surface $S_{K}$ to one side. This almost normal surface is then isotopic to a normal surface by Lemma 15 and Lemma 16 which we will call $\Gamma_{i}^{\text {top }}$ (or $\Gamma_{i}^{\text {bot }}$ depending on whether we compressed $S_{K}$ above or below). The surface $\Gamma_{i}^{\text {top }}$ determines a submanifold $M_{i}$ of $M_{0}$ with $\Gamma_{i}^{\text {top }} \subset \partial M_{i}$. The surface $S_{K}$ separates $M_{i}$ into two $K_{i}$-compression bodies, $W_{i}$ and $W_{i}^{\prime}$ with $\partial_{-} W_{i}=\Gamma_{i}^{\text {top }}$ and $\partial_{-} W_{i}^{\prime}=\Gamma_{i}^{\text {bot }}$. The spines of these $K_{i}-$ compression bodies are now what we will call $\Sigma_{i}^{\text {top }}$ and $\Sigma_{i}^{\text {bot }}$ respectively.

Denote by $t_{i}^{\text {top }}$ and $t_{i}^{\text {bot }}$ the boundary of a regular neighborhood $N\left(\Sigma_{i}\right)$ in $M_{i}$ which we continue to call "tubes". A regular leaf near the top (resp. bottom) singular leaf is then obtained by attaching $t_{i}^{\text {top }}\left(\right.$ resp. $\left.t_{i}^{\text {bot }}\right)$ to a punctured copy of $\Gamma_{i}^{\text {top }}\left(\operatorname{resp} . \Gamma_{i}^{\text {bot }}\right)$.

The surface $S_{K}$ is a weakly incompressible splitting surface for $M_{i}$ that separates it into two $K$-compression bodies $W_{i}$ and $W_{i}^{\prime}$ for each $i \geq 0$. To see this observe that if $S_{K}$ is a weakly incompressible splitting surface for $M_{i-1}$ then since $M_{i}$ was constructed by compressing along disks in $W_{i-1}$ and $W_{i-1}^{\prime}$ to obtain $W_{i}$ and $W_{i}^{\prime}$ it follows that the collection of compressing disks in $W_{i}$ and $W_{i}^{\prime}$ are a subset of the collection of compressing disks in $W_{i-1}$ and $W_{i-1}^{\prime}$. Since every pair of compressing disks on opposite sides of $S_{K}$ intersect in $M_{i-1}$ it follows that every pair of compressing disks on opposite sides of $S_{K}$ in $M_{i}$ must intersect as well.

Here is a sketch of the iterative process that we will describe in detail later. If it happens that at the first step of this process the surface $S_{K}$ is isotopic to the collection of annuli $\Gamma_{0}$ then we can conclude that $K$ is the unknot. Otherwise, start with $\left(M_{i}, \Sigma_{i}, \Gamma_{i}\right)$. If, without loss of generality, $\chi\left(\Gamma_{i}^{\text {top }}\right)=\chi\left(S_{K}\right)$ and $\Gamma_{i}^{\text {top }}$ is isotopic to an almost normal surface then since $\Gamma_{i}^{\text {top }}$ is obtained by compressing a leaf of the foliation it follows that $\Gamma_{i}^{\text {top }}$ is isotopic to $S_{K}$ and we are done. Otherwise apply either Lemma 17 or Lemma 24 to obtain a new collection of normal and almost normal surfaces in $M_{i}$. If there is 
an almost normal surface $G$ in the collection with $\chi(G)=\chi\left(S_{K}\right)$ then again because the almost normal surface comes from compressing a leaf of the foliation we know $G$ must be isotopic to $S_{K}$ and we are done. Otherwise since by Lemma 16 the surface $G$ is incompressible to one side we can use Lemma 15 to isotope the almost normal surfaces (if any) in the collection to be normal. This collection of normal surfaces now becomes either $\Gamma_{i+1}^{\text {top }}$ or $\Gamma_{i+1}^{\text {bot }}$, depending on whether we compressed $S_{K}$ above or below. Then, using this new collection of normal surfaces we can cut $\left(M_{i}, \Sigma_{i}, \Gamma_{i}\right)$ along this collection to obtain $\left(M_{i+1}, \Sigma_{i+1}, \Gamma_{i+1}\right)$, which will also satisfy the above properties. It turns out that we only need to repeat the recursive step a finite number of times before obtaining an almost normal surface isotopic to $S_{K}$. This completes the sketch.

Now, consider the $\operatorname{arcs} \mathcal{T}_{i}^{1}$ in $M_{i}$ in thin position with respect to $F_{i}$. Recall that all ends of $\mathcal{T}_{i}^{1}$ lie on $\Gamma_{i}^{\text {top }}$ or $\Gamma_{i}^{\text {bot }}$, part of the top or bottom singular leaves of $F_{i}$. One possibility is that there is a maximum of $\mathcal{T}_{i}^{1}$ that is above a minimum of $\mathcal{T}_{i}{ }^{1}$ which implies that there is a thick region of $\mathcal{T}_{i}^{1}$ in $M_{i}$. The other possibility is that all of the minima of $\mathcal{T}_{i}^{1}$ are above all of the maxima of $\mathcal{T}_{i}^{1}$ and so there is no thick region. In this situation we will consider separately the following two possibilities. The first is that there is no thick region and there is some arc of $\mathcal{T}_{i}^{1}$ with both ends on $\Gamma_{i}^{\text {top }}$ or both ends on $\Gamma_{i}^{\text {bot }}$. The second possibility is that there is no thick region and each arc each arc of $\mathcal{T}_{i}^{1}$ has one endpoint on $\Gamma_{i}^{\text {top }}$ and the other endpoint on $\Gamma_{i}^{\text {bot }}$. We will consider each of the three possibilities in turn.

The first possibility is that there a thick region of $\mathcal{T}_{i}^{1}$ with respect to $F_{i}$.

Lemma 17 ( $\mathrm{cf}\left[14\right.$, Lemma 5]) If there is a thick region for $\mathcal{T}_{i}^{1}$ in $M_{i}$, then there is a collection of normal and almost normal surfaces in $M_{i}$ obtained from a leaf of the foliation by compressing the leaf to one side. At most one surface in the collection can be almost normal. Not all of the surfaces are boundary parallel.

Proof of Lemma 17 The proofs of Claim 18, Claim 19, Claim 20 and Claim 21 can be found in [15]. Let $\left(M_{i}, \Sigma_{i}, \Gamma_{i}\right)$ be as described above, where $\mathcal{T}_{i}^{1}$ is in thin position with respect to the foliation $F_{i}$ of $M_{i}$. Since there is a thick region of $\mathcal{T}_{i}^{1}$ in $F_{i}$ we can apply [15, Claim 4.5].

Claim 18 ([15, Claim 4.5]) There exists a transverse leaf $L$ in the first thick region of $F_{i}$ which intersects the 2-skeleton entirely in normal arcs and simple closed curves disjoint from the 1-skeleton.

Let $L$ be a leaf of $F_{i}$ in a thick region intersecting the 2 -skeleton in normal arcs and simple closed curves disjoint from $\mathcal{T}^{1}$ as is guaranteed by Claim 18. Then we can apply Claim 19, Claim 20 and Claim 21 to the leaf $L$. 
Claim 19 ([15, Claim 4.1]) Let $H$ be any tetrahedron in the triangulation $\mathcal{T}$ of $M_{K}$. Then $L \cap \partial H$ contains no parallel curves of length greater than or equal to eight.

Claim 20 ([15, Claim 4.2]) Let $H$ be any tetrahedron in the triangulation $\mathcal{T}$ of $M_{K}$. Then $L \cap \partial H$ contains no curve of length greater than eight.

Claim 21 ([15, Claim 4.3]) Let $H_{1}$ and $H_{2}$ be distinct tetrahedra in the triangulation of $M_{K}$. Then $L \cap \partial H_{1}$ and $L \cap \partial H_{2}$ do not both contain curves of length eight.

The above claims together imply that this leaf $L$ of the foliation $F_{i}$ intersects the 2 -skeleton only in simple closed curves disjoint from the 1-skeleton and normal curves of lengths three, four and at most one of length eight. Compressing the simple closed curves in $L \cap \mathcal{T}^{2}$ as well as any compressions in the interior of the tetrahedra gives a collection of normal surfaces with at most one almost normal surface. The almost normal surface, if it exists, must be a normal octagon since we have compressed any almost normal annuli in the interior of the tetrahedra. We can think of the leaf $L$ as this collection of normal and almost normal surfaces with tubes attached. Since our triangulation has no normal 2-spheres we can conclude that this collection will contain no almost normal 2-spheres as well since any almost normal 2-sphere can be isotoped to give a normal one by Lemma 15 . Also notice that in this collection we will not have a normal surface with a tube attached to the side opposite the side that we compressed to or we get a contradiction to the leaf being weakly incompressible. As long as there are no normal 2-spheres in this collection of normal surfaces then the collection of disks of $L \cap \partial H$ that arise in each tetrahedron $H$ of $\mathcal{T}$ correspond to actual compressing disks of $L$. Also, since this collection of disks is disjoint these compression disks must all be to the same side of $L$ otherwise we again contradict the fact that the surface $L$ is weakly incompressible. In general, by Lemma 16 compressing $L$ to one side gives a surface that is incompressible to the opposite side. Therefore all compressions must be to one side of $L$. This completes the proof of Lemma 17.

Remark 22 There is no choice in the direction in which the tubes of $L$ compress, however Lemma 16 implies that after compressing $L$, the remaining collection of normal and almost normal surfaces is incompressible in the direction opposite to which we have compressed.

For the proof of the Lemma 24 we will need the following claim.

Claim 23 A properly embedded, orientable, normal surface is incompressible and boundary incompressible in the complement of the 1 -skeleton $\mathcal{T}^{1}$. 
Proof This claim follows from a standard innermost disk and outermost arc argument.

The second possibility is that there is no thick region and some arc of $\mathcal{T}_{i}^{1}$ has both endpoints on $\Gamma_{i}^{\text {top }}$ or both endpoints on $\Gamma_{i}^{\text {bot }}$.

Lemma 24 (cf [14, Lemma 4]) If there is no thick region for $\mathcal{T}_{i}^{1}$ in $M_{i}$ and some arc of $\mathcal{T}_{i}^{1}$ has both endpoints on $\Gamma_{i}^{\text {top }}$ (resp. has both endpoints on $\Gamma_{i}^{\text {bot }}$ ), then there is an almost normal surface in $M_{i}$ that is isotopic to a surface obtained from a leaf of $F_{i}$ by compressing the leaf above (resp. below).

Proof We will prove the Lemma for arcs of $\mathcal{T}_{i}^{1}$ with both endpoints on $\Gamma_{i}^{\text {top }}$. The argument for arcs of $\mathcal{T}_{i}^{1}$ with both endpoints on $\Gamma_{i}^{\text {bot }}$ is symmetric. Let $L$ be a leaf of the foliation near the top singular leaf $\Gamma_{i}^{\text {top }} \cup \Sigma_{i}^{\text {top }}$, so that $L$ consists of the normal surface $\Gamma_{i}^{\text {top }}$ punctured and attached to the tubes $t_{i}^{\text {top }}=\partial N\left(\Sigma_{i}^{\text {top }}\right)$. See Figure 1 for the case $i=0$ and $M=S^{3}$. Let $\beta$ be an arc of $\mathcal{T}_{i}^{1}$ with both endpoints on $\Gamma_{i}^{\mathrm{top}}$. Since there is no thick region for $\mathcal{T}_{i}^{1}, \beta$ has only a single minimum and it is parallel to an $\operatorname{arc}$ on $L$, so there is a lower disk $E$ whose boundary is the union of $\beta$ and an $\operatorname{arc} \alpha$ in $L$. We will show that after some edge slides and isotopies of $\Sigma_{i}^{\text {top }}, \alpha$ runs once over exactly one tube of $t_{i}^{\text {top }}$ and that this tube connects two normal disks in a tetrahedron, therefore is part of an almost normal surface.

Define the complexity of $E$ to be $(a, b)$, lexicographically ordered, where $a$ is the number of points of $\Sigma_{i}^{\text {top }} \cap \mathcal{T}^{2}$ to which $\alpha$ is also incident and $b$ is the number of components in which $E$ meets the 2-skeleton of $\mathcal{T}$. We will assume that the complexity of $E$ has been minimized over all choices of $E$.

Observe that the arc $\alpha$ of $\partial E$ can't lie entirely in $\Gamma_{i}^{\text {top }}$. Otherwise $E$ would be a boundary compressing disk in the complement of the 1-skeleton of the normal surface $\Gamma_{i}^{\text {top }}$ which contradicts Claim 23. Our strategy will be to show that there is a sequence of proper isotopies of $\Sigma_{i}^{\text {top }}$ and slides of ends of arcs of $\Sigma_{i}^{\text {top }}$ over each other and over $\Gamma_{i}^{\text {top }}$ (neither of which affect the isotopy class of $\Gamma_{i}^{\text {top }} \cup \Sigma_{i}^{\text {top }}$ ) so that afterwards $\alpha$ is incident to a single edge $z$ in $\Sigma_{i}^{\text {top }}, \alpha$ runs along this edge once and $E$ lies entirely inside a single tetrahedron. Then $\Gamma_{i}^{\text {top }} \cup \partial(N(z))$ is the required almost normal surface obtained from $L$ by compressing all other tubes of $\Sigma_{i}^{\text {top }}$. Now that we have established some notation, for the rest of the proof we will consider the intersections of the disk $E$ with the 2 -skeleton of $\mathcal{T}$ and we will show that any intersection violates the minimality of $(a, b)$.

When we consider the arcs of intersection between $E$ and the 2-skeleton, we can get four types of components of $E \cap \mathcal{T}^{2}$ in $E$. See Figure 3. Components of Type I are 
simple closed curves in $E$. Components of Type II are arcs with both endpoints on $\alpha$. Components of Type III are arcs with both endpoints on $\beta$ and components of Type IV are arcs with one endpoint on $\alpha$ and the other endpoint on $\beta$. Next we will describe how each type of component of intersection of $E \cap \mathcal{T}^{2}$ can be removed, violating minimality of $(a, b)$.

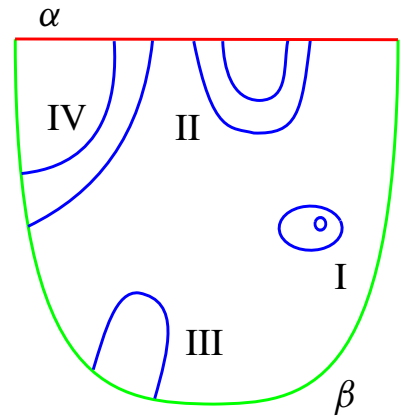

Figure 3: The disk E

Removing components of Type I Components of Type I are simple closed curves in $E$. A component of Type I that is innermost in a 2-simplex of $\mathcal{T}^{2}$ can be removed by substituting the disk it bounds in $\mathcal{T}^{2}$ for the disk it bounds in $E$. This reduces the number of times that $E$ meets the 2-skeleton, thus reducing $b$ and contradicting the minimality of $E$.

Removing components of Type II A component of Type II corresponds to an arc in a face $\sigma$ of some tetrahedron of $\mathcal{T}$ that either has both endpoints on distinct components of $\left(\Sigma_{i}^{\text {top }} \cup \Gamma_{i}^{\text {top }}\right) \cap \sigma$, or has both endpoints on the same component of $\left(\Sigma_{i}^{\text {top }} \cup \Gamma_{i}^{\text {top }}\right) \cap \sigma$. Suppose $\gamma$ is an arc of intersection between $\mathcal{T}^{2}$ and $E$ that is outermost in $E$ and is of Type II. See Figure 4(a). Let $\delta$ be a subarc of $\alpha$ such that $\gamma \cup \delta$ is the boundary of a disk $D$ in $E-\mathcal{T}^{2}$. In what follows we will use edge slides of $\Sigma_{i}^{\text {top }}$ to remove components of $\mathcal{T}^{2} \cap E$ and $\mathcal{T}^{2} \cap \Sigma_{i}^{\text {top }}$. Recall that $t_{i}^{\text {top }}$ is the boundary of a neighborhood of $\Sigma_{i}^{\text {top }}$ and we will abuse notation and consider $\delta \subset \alpha$ as an $\operatorname{arc}$ on $\Sigma_{i}^{\text {top }}$ when we really mean that $\delta$ is an arc on $t_{i}^{\text {top }}$.

Any two ends of edges of $\Sigma_{i}^{\text {top }}$ that meet the same normal disk in $\Gamma_{i}^{\text {top }}$ can be isotoped together so that there is at most one edge incident to each normal disk. Since $\Sigma_{i}^{\text {top }}$ can be extended to give a spine of $W$, any cycle in $\Sigma_{i}^{\text {top }}$ gives a cycle in $\Sigma$. Since $W \cup_{S} W^{\prime}$ is an irreducible Heegaard splitting of $M$ it follows from Frohman [5] (also see [12, Proposition 2.5]) that no cycle of $\Sigma_{(W, K)}$ lies in a 3-ball. Hence for any tetrahedron $H$ of $\mathcal{T}, \Sigma_{i}^{\text {top }} \cap H$ cannot contain a cycle. Thus $\Sigma_{i}^{\text {top }} \cap H$ is a union 


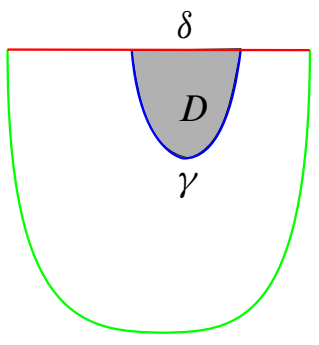

(a)

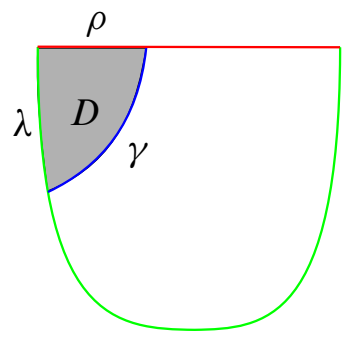

(b)

Figure 4: Arcs of Type II and IV in $E$

of trees for each tetrahedron $H$ in $\mathcal{T}$. Each component of $\Sigma_{i}^{\text {top }} \cap H$ is a tree and each component of $\left(\Sigma_{i}^{\text {top }} \cup \Gamma_{i}^{\text {top }}\right) \cap H$ is a tree with disks attached and so is simply connected.

Case 1 The arc $\gamma$ has both endpoints on distinct components of $\left(\Sigma_{i}^{\mathrm{top}} \cup \Gamma_{i}^{\mathrm{top}}\right) \cap \sigma$.

Case 1.1 Let $H$ be the tetrahedron in $\mathcal{T}$ that contains $\delta$ and let $q$ denote the component of $\Sigma_{i}^{\text {top }} \cap H$ that contains $\delta$. If $q$ is a single arc with both endpoints on the same face of $H$ then $D$ describes an isotopy that removes two points of intersection of $q$ with $\mathcal{T}^{2}$. This reduces the number of points of $\Sigma_{i}^{\text {top }} \cap \mathcal{T}^{2}$ to which $\alpha$ is incident, thus reducing $a$, which is a contradiction.

Case 1.2 Now suppose that $q$ is not an arc and $\delta$ is a path in $q$ that begins at point $x$ in $q$ that is not in a normal disk but is in some face $\sigma$ of $H$. See Figure 5. Let $z$ be the edge of $\Sigma_{i}^{\text {top }}$ containing $x$. Then $\delta$ describes a series of edge slides of $z$ which culminate by introducing an extra point of intersection between $\Sigma_{i}^{\text {top }}$ and $\sigma$. However, after the edge slides the disk $D$ runs only over the edge $z$. Hence we can reduce the number of intersections of edges of $\Sigma_{i}^{\text {top }}$ incident to $\alpha$ that meet $\mathcal{T}^{2}$ by two as in Case 1.1 reducing $a$ by at least one and contradicting the minimality assumptions.

Case 1.3 If $\delta$ is an arc on $L$ that has both endpoints of $\mathcal{T}^{2} \cap E$ on normal disks of $L$, then either $\delta$ must run over some edges of $\Sigma_{i}^{\text {top }}$ or it lies in a normal disk. If $\delta$ lies on a normal disk in $H$ then it bounds a subdisk $D^{\prime}$ of the normal disk. Together $D$ and $D^{\prime}$ bound a 3-ball in $H$ that can be used to isotope $E$ into the next tetrahedron removing $\gamma$ and reducing complexity.

So we can assume that $\delta$ runs over some edges of $\Sigma_{i}^{\text {top }}$. Say that $\delta$ runs from normal disk $D_{1}$ to normal disk $D_{2}$. Since $\Sigma_{i}^{\text {top }}$ is incident to $D_{1}$ in only a single point, $\delta$ is incident to $\partial D_{1}$ in a single point. It follows that $D_{1}-N\left(\Sigma_{i}^{\text {top }}\right)$ is an annulus and $\delta$ intersects the annulus in a single spanning arc. Thus $\delta$ runs precisely once along the 

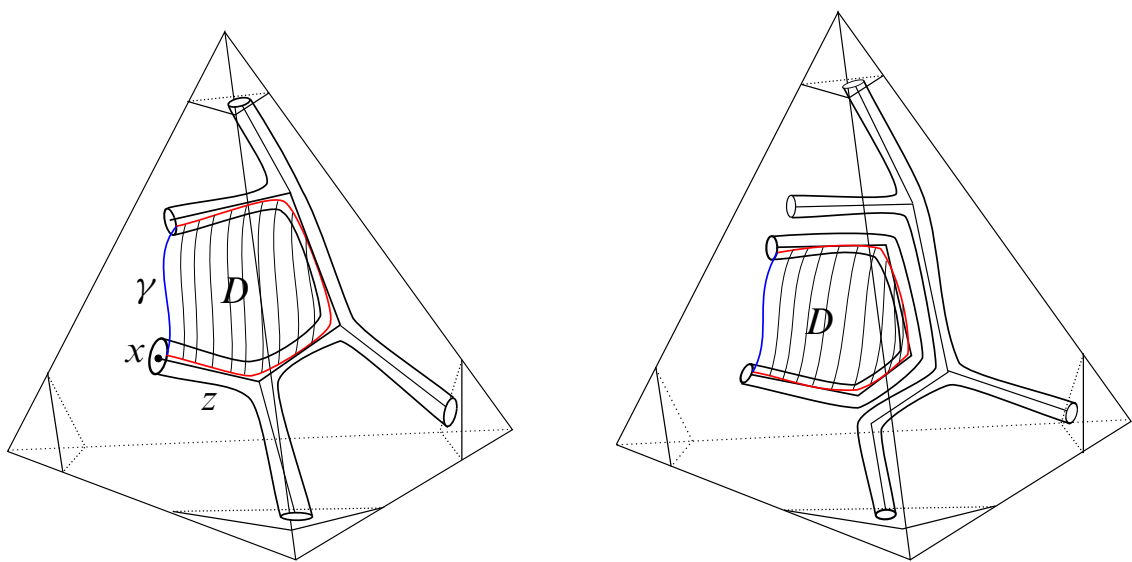

Figure 5

edge $z$ that is incident to $D_{1}$. Then, as above, $D$ describes a slide and isotopy of $z$ that carries it to the arc $\gamma$ in a simplex of $\mathcal{T}^{2}$. But then a subdisk of that face describes a parallelism between $z$ and a subarc of $\mathcal{T}^{1}$. In particular, attaching a tube to $\Gamma_{i}^{\text {top }}$ along $z$ gives an almost normal surface.

Case 2 The $\operatorname{arc} \gamma$ has both endpoints on the same component of $\left(\Sigma_{i}^{\text {top }} \cup \Gamma_{i}^{\text {top }}\right) \cap \sigma$.

In this case the arc $\gamma$ has both endpoints on the same component of $q \cap \partial H$. Let $x$ denote the endpoint of $q$ in the face $\sigma$ of tetrahedron $H$. We assume that $x \in \Sigma_{i}^{\text {top }}$, so $\gamma$ forms a loop based at $x$ in $\sigma$ bounding a disk $A$ in $\sigma$; the case where both ends of $\gamma$ lie on a normal disk is similar.

Case 2.1 If $\operatorname{int}(A) \cap \Sigma_{i}^{\text {top }}=\varnothing$, then construct new disks $E^{\prime}$ and $E^{\prime \prime}$ by cutting the disk $D$ along $\gamma$ and attaching a copy of $A$ to each piece. One of the disks $E^{\prime}$ or $E^{\prime \prime}$ will still be a lower disk and it will meet $\mathcal{T}^{2}$ in fewer components than $E$, contradicting the minimality of $E$.

Case 2.2 Now suppose that $A \cap \Sigma_{i}^{\text {top }} \neq \varnothing$. Since $\Sigma_{i}^{\text {top }}$ is a union of trees in $H$, we know that a neighborhood of each component $q$ of $\Sigma_{i}^{\text {top }} \cap H$ is a 3-ball. So there is a disk $D^{\prime}$ in $N(q)$ whose boundary is the union of $\delta$ and a diameter $\delta^{\prime}$ of a small disk $\epsilon$ with which $N(q)$ meets $\partial H$ at $x$.

Isotope the leaf $L$ by compressing $\delta$ to $\delta^{\prime}$ via the disk $D^{\prime}$ in $N(q)$, splitting the disk $\epsilon$ in two. See Figure 6. The effect on the spine is a possibly complicated series of edge slides. The overall effect is that the number of components of $\Sigma_{i}^{\text {top }} \cap \sigma$ increases by one when $\epsilon$ splits and $D \cup D^{\prime}$ becomes a disk disjoint from $\Sigma_{i}^{\text {top }}$ and parallel to $A$. 
The disk $A$ now contains at least two points of $\Sigma_{i}^{\text {top }} \cap \sigma$. Now push $D \cup D^{\prime}$ across $A$ to remove $\gamma$, thus reducing $b$ which is a contradiction.
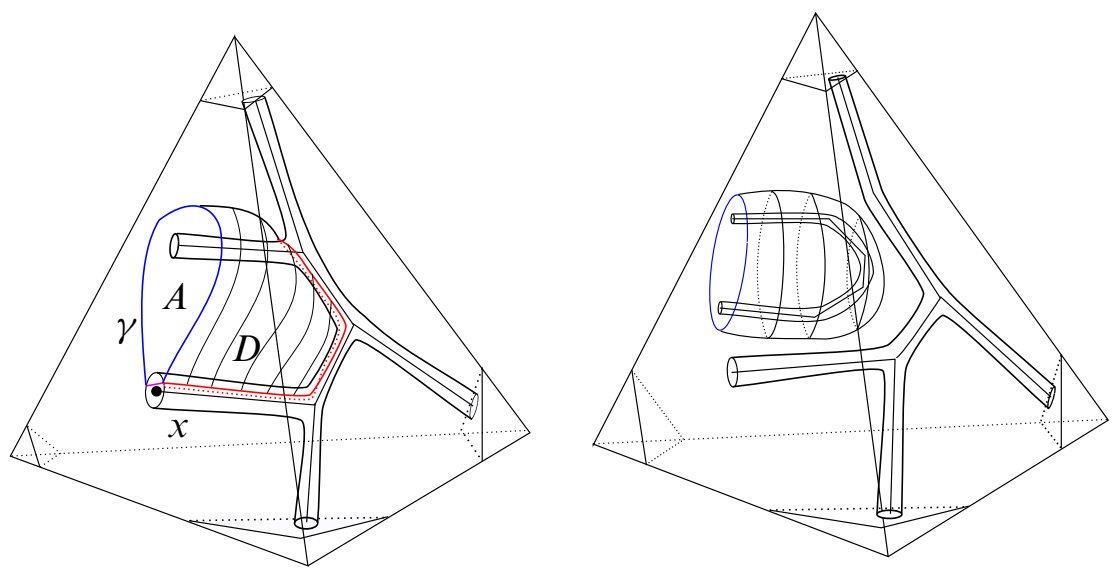

Figure 6

Removing components of Type III and IV To see how to remove components of Types III and IV it will be helpful to view the arc $\beta$ that runs along the edge $e$ of $\mathcal{T}^{1}$ as an arc that lies on $\partial N(e)$. As an $\operatorname{arc}$ on $\partial N(e), \beta$ may wind around the edge $e$. If the winding is not monotone a priori then we can reduce the number of components in which the disk $E$ meets the faces of $\mathcal{T}^{2}$, contradicting minimality. Thus we may assume that the curve $\beta$ winds monotonically around the edge $e$, creating a "barber pole" effect shown in Figure 7 and Figure 8. This implies that there are no curves of Type III since the existence a curve of Type III means that the arc $\beta$ must 'double back' as it winds around $e$, contradicting monotonicity.

Let $\gamma$ be an outermost arc component of Type IV, and let $D$ be the corresponding outermost sub-disk of $E$. Let $\sigma$ be the face of $\mathcal{T}^{2}$ that contains $\gamma$. See Figure 4(b). The disk $D$ is co-bounded by a sub-arc $\rho$ of $\alpha$, a sub-arc $\lambda$ of $\beta$, and $\gamma$. Let $H$ denote the tetrahedron containing $D$ in its interior and with $\sigma$ as a face.

There are two cases that we will consider separately. The first case is when the arc $\gamma$ of $E \cap \sigma$ that runs from the edge $e$ to $L$ ends on a normal disk $\eta$ of $L$. The second case is when $\gamma$ ends on a tube (neighborhood of $\Sigma_{i}^{\text {top }}$ ) of $L$.

Case 3.1 Suppose first that $\gamma$ ends on a normal disk $\eta$. See Figure 7. In this situation there are two subcases. Either $\Sigma_{i}^{\mathrm{top}} \cap \rho=\varnothing$ or $\Sigma_{i}^{\mathrm{top}} \cap \rho \neq \varnothing$.

Case 3.1.1 Suppose that $\Sigma_{i}^{\text {top }} \cap \rho=\varnothing$. In this case the arc $\rho$ runs over only normal disks and does not meet any tubes of $\Sigma_{i}$. See Figure 7(a). Observe that there is a disk 
$D^{\prime}$ in $\partial N(e)$ whose boundary consists of 3 arcs. The first arc is the subarc $\lambda$ of $\beta$. The second arc is an arc on $\partial N(e)$ that corresponds to where the face $\sigma$ meets $\partial N(e)$. The third arc is a subarc of a meridian of $\partial N(e)$ that corresponds to where $\eta$ meets $\partial N(e)$. In this case $D^{\prime} \cup \eta \cup D \cup \sigma$ bounds a 3-ball in $H$ that we can use to isotope $D$ across $\sigma$ and into the next tetrahedron. If no components of $\Sigma_{i}^{\text {top }}$ are contained in the 3-ball then isotoping $D$ across $\sigma$ and into the next tetrahedron will remove $\gamma$ and reduce $b$, thus reducing the complexity of $E$, giving a contradiction. If components of $\Sigma_{i}^{\text {top }}$ are contained in the 3-ball then the overall effect of isotoping $D$ into the next tetrahedron will remove $\gamma$ thus reducing $b$ and will not increase the number of components of $\Sigma_{i}^{\text {top }} \cap \mathcal{T}^{2}$, and again the complexity of $E$ has been reduced giving a contradiction.

Case 3.1.2 Suppose now that $\Sigma_{i}^{\text {top }} \cap \rho \neq \varnothing$. See Figure 7(b). Since $\Sigma_{i}^{\text {top }}$ is a union of trees, each component of $N\left(\Sigma_{i}^{\text {top }}\right)$ is a 3-ball. In particular, there is a disk $\Delta$ in $N\left(\Sigma_{i}^{\text {top }}\right)$ whose boundary is the union of a sub-arc of $\rho$ and a diameter $d$ of the disk $\epsilon$ with which $N\left(\Sigma_{i}^{\text {top }}\right)$ intersects the normal disk $\eta$. Isotope $N\left(\Sigma_{i}^{\text {top }}\right)$ by compressing $d$ to $\rho$ in $N\left(\Sigma_{i}^{\text {top }}\right)$, splitting the disk $\epsilon$ in two. The effect on $\Sigma_{i}^{\text {top }}$ is a series of edge slides that results in a new component of $\Sigma_{i}^{\text {top }} \cap H$ that is on the same side of $\rho$ as $e$. We can repeat this process until we have removed all components of $\Sigma_{i}^{\text {top }} \cap \rho$. Now proceed as in Case 3.1.1.

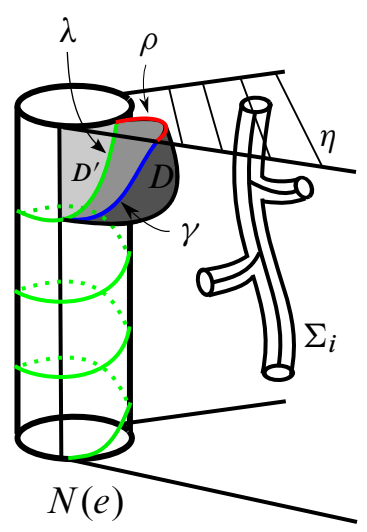

(a)

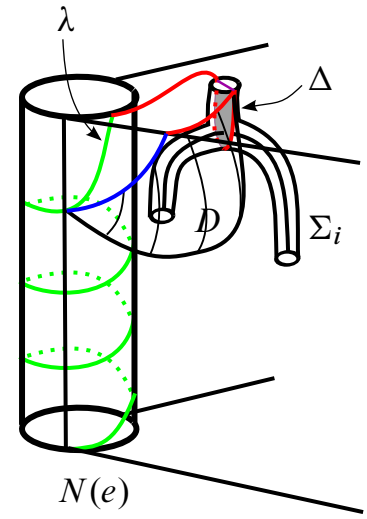

(b)

Figure 7: Arcs of type IV

Case 3.2 Now suppose that $\gamma$ ends on a tube of $L$. See Figure 8. The core of this tube is an edge $\tau$ that may connect to other edges of $\Sigma_{i}^{\text {top }}$ in $\Sigma_{i}^{\text {top }} \cap H$, and $\Sigma_{i}^{\text {top }}$ connects to a normal disk $\eta$. We will describe in two steps a slide of $\tau$ and an isotopy 
of $D$ that will remove a component of $\Sigma_{i}^{\text {top }} \cap E$, reducing $a$, and thereby reducing the complexity of $E$.

First, since $\tau$ connects to other edges of $\Sigma_{i}$ in $\Sigma_{i} \cap H, \rho$ describes an edge slide of $\tau$ that keeps $\tau \cap \sigma$ fixed but slides the opposite end of the edge $\tau$ off of $\Sigma_{i}$ and onto the normal disk $\eta$. See Figure 8(b). We continue to slide $\tau$ along $\eta$ following $\rho$ until it almost meets $\partial N(e)$. Now we can use the disk $D$ to isotope all of $\tau$ until it lies close to $\lambda \cup \gamma$. See Figure 8(c). At this point the entire disk $D$ and tube $\tau$ lie very close to $\beta \cup \gamma$ in $H$.

For the second step recall the disk $D^{\prime}$ in $\partial N(e)$ that is bounded by $\lambda$, a copy of part of the edge $e$ that bounds the face $\sigma$ and a copy of a meridian of $\partial N(e)$. Together the disks $D$ and $D^{\prime}$ describe an isotopy of $\tau$ across the face $\sigma$ and into the next tetrahedron, removing the component $\gamma$ from $\sigma \cap E$ and, in particular, removing the component of intersection between $\tau$ and $\sigma$ in $\mathcal{T}^{2}$, reducing $a$, which is a contradiction. See Figure 8(d). Thus there can be no arcs of Type IV. Therefore the arc $\beta$ of $\mathcal{T}_{i}{ }^{1}$, the edges of $\Sigma_{i}^{\text {top }}$ that $\alpha$ runs along and the disk $E$ are all contained in one tetrahedron. An argument similar to the one given in the last paragraph of Case 1 above shows that $\alpha$ is incident to a single edge in $\Sigma_{i}^{\text {top }}$ and $\alpha$ runs along this edge exactly once. Attaching a tube to $\Gamma_{i}^{\text {top }}$ along this edge and compressing all other tubes of $\Sigma_{i}^{\text {top }}$ in $L$ gives an almost normal surface. By Lemma 16 compressing $L$ to one side gives a surface that is incompressible to the opposite side. Therefore all compressions must be to one side of $L$, namely all compressions are either above or below.

The third possibility is that there is no thick region and each arc of $\mathcal{T}_{i}{ }^{1}$ has one endpoint on $\Gamma_{i}^{\text {top }}$ and the other endpoint on $\Gamma_{i}^{\text {bot }}$.

Lemma 25 If there is no thick region for $\mathcal{T}_{i}^{1}$ in $M_{i}$ and each arc of $\mathcal{T}_{i}^{1}$ has one endpoint on $\Gamma_{i}^{\text {top }}$ and has the other endpoint on $\Gamma_{i}^{\text {bot }}$ then $M_{i}$ is a product region.

Proof Recall that $\partial M_{i}=\Gamma_{i}^{\mathrm{top}} \cup \Gamma_{i}^{\mathrm{bot}}$. Since $\Gamma_{i}^{\mathrm{top}}$ and $\Gamma_{i}^{\mathrm{bot}}$ are normal with respect to $\mathcal{T}$ it follows that there are two possibilities for how the region $M_{i}$ between $\Gamma_{i}^{\text {top }}$ and $\Gamma_{i}^{\text {bot }}$ can intersect a face of the 2 -skeleton. Either the region bounded by $\Gamma_{i}^{\text {top }}$ and $\Gamma_{i}^{\text {bot }}$ is a trapezoid region or a hexagon region. See Figure 9.

Suppose that there is a hexagon region of $M_{i} \cap \mathcal{T}^{2}$. Then three edges of the hexagon are arcs of $\left(\Gamma_{i}^{\text {top }} \cup \Gamma_{i}^{\text {bot }}\right) \cap \mathcal{T}^{2}$ and the other three edges are arcs of $\mathcal{T}_{i}^{1}$ connecting the three components of $\Gamma_{i} \cap \mathcal{T}^{2}$. But this implies that some arc of $\mathcal{T}_{i}^{1}$ connects either $\Gamma_{i}^{\text {top }}$ to $\Gamma_{i}^{\text {top }}$ or $\Gamma_{i}^{\text {bot }}$ to $\Gamma_{i}^{\text {bot }}$ which is a contradiction. Therefore there cannot be any hexagonal components and all regions of intersection between $M_{i}$ and $\mathcal{T}^{2}$ are trapezoids. 


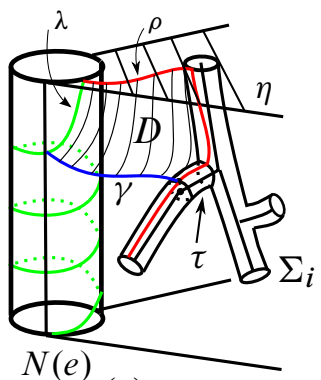

(a)

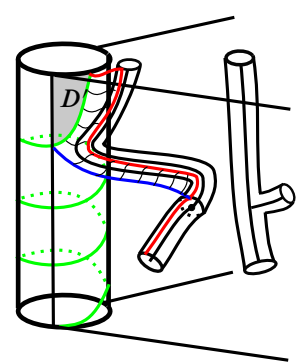

(c)

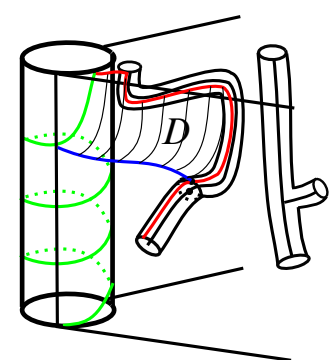

(b)

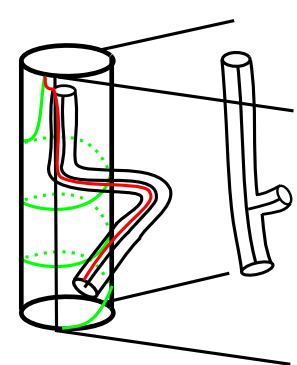

(d)

Figure 8: Arcs of type IV

Because we know that there are no hexagonal components of intersection between $M_{i}$ and $\mathcal{T}^{2}$ this implies that the only possibilities for components of intersection between $M_{i}$ and the tetrahedra of the 3-skeleton are triangular product regions and quadrilateral product regions. Each triangular and quadrilateral product region is bounded on one side by a normal disk of $\Gamma_{i}^{\mathrm{top}}$ and on the other by a normal disk of $\Gamma_{i}^{\text {bot }}$. Since each component of $M_{i} \cap H$ is a product region with one end on each of $\Gamma_{i}^{\text {top }}$ and $\Gamma_{i}^{\text {bot }}$ for each tetrahedron $H$ in $\mathcal{T}$ we can conclude that $M_{i}$ is itself such a product region. $\square$

We can now complete the proof of Main Theorem 1. We will prove the theorem by describing a recursive process that will end when it produces an almost normal surface isotopic to the bridge surface $S_{K}$. Recall that we began with a knot $K$ in a closed 3-manifold $M$ with the assumptions that $M$ and $M_{K}$ are irreducible. We foliated $M_{K}$ by copies of the bridge surface $S_{K}$ with two singular leaves and triangulated $M_{K}$ so that the annuli $\Gamma$ are normal and the vertices of $\mathcal{T}$ are to one side of $\Gamma$. Cutting along a maximal family of non-parallel normal 2-spheres tubed to the normal annuli $\Gamma$ we obtained the submanifold $M_{0}$ of $M_{K}$. The surface $S_{K}$ induces a splitting of $M_{0}$ into $K_{0}$-compression bodies $W_{0}$ and $W_{0}^{\prime}$ and $M_{0}$ is foliated by copies of the bridge surface $S_{K}$ and where the top (resp. bottom) leaf of the foliation is given by 

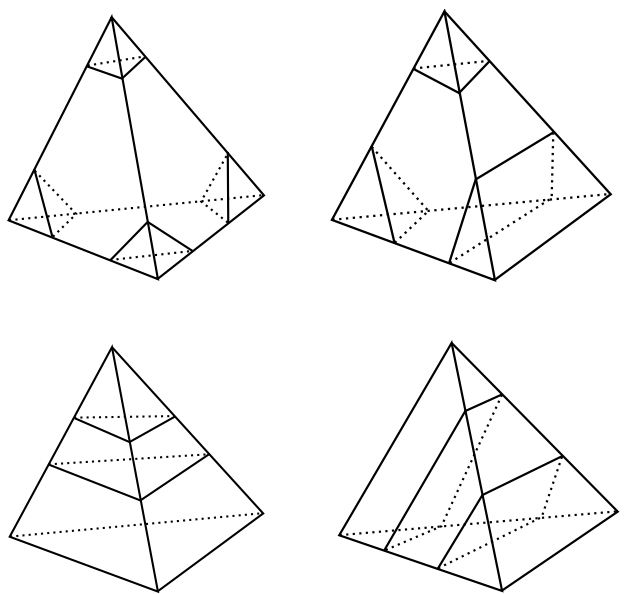

Figure 9

the union of the spine $\Sigma_{0}^{\text {top }}$ (resp. $\Sigma_{0}^{\text {bot }}$ ) of $W_{0}$ (resp. $W_{0}^{\prime}$ ) and $\Gamma_{0}^{\text {top }}$ (resp. $\Gamma_{0}^{\text {bot }}$ ). Here $\Gamma_{0}^{\text {top }}=\Gamma^{\text {top }}$ (resp. $\Gamma_{0}^{\text {bot }}=\Gamma^{\text {bot' }}$ ) are the normal annuli in $\partial M_{0}$. The triple $\left(M_{0}, \Sigma_{0}, \Gamma_{0}\right)$ is the beginning of the recursive process. Each later step will produce a triple $\left(M_{i}, \Sigma_{i}, \Gamma_{i}\right)$ such that $M_{i} \subset M_{i-1}$ and for each $i$ the surface $S_{K}$ is a weakly incompressible splitting surface for $M_{i}$ separating it into two $K_{i}$-compression bodies $W_{i}$ and $W_{i}^{\prime}$, where $K_{i}=K \cap M_{i}$. The spine $\Sigma_{i}^{\text {top }}$ (resp. $\Sigma_{i}^{\text {bot }}$ ) of $W_{i}$ (resp. $W_{i}^{\prime}$ ) is contained in some spine for $W$ (resp. $W^{\prime}$ ) and $\Gamma_{i}=\partial M_{i}-\partial M$ is a pair of collections of normal surfaces $\Gamma_{i}^{\text {top }}$ and $\Gamma_{i}^{\text {bot }}$ in $\partial M_{i}$.

The top (bottom) leaf of a singular foliation $F_{i}$ is given by the union of $\Gamma_{i}^{\text {top }}$ (resp. $\Gamma_{i}^{\text {bot }}$ ) and the intersection $\Sigma_{i}^{\text {top }} \subset \Sigma^{\text {top }}$ with $M_{i}$ (resp. $\Sigma_{i}^{\text {bot }} \subset \Sigma^{\text {bot }}$ with $M_{i}$ ). Put $\mathcal{T}_{i}^{1}$, the part of $\mathcal{T}^{1}$ lying in $M_{i}-\partial M$, in thin position with respect to $F_{i}$. As mentioned earlier, either the arcs of $\mathcal{T}_{i}^{1}$ all have one endpoint on $\Gamma_{i}^{\text {top }}$ and one endpoint on $\Gamma_{i}^{\text {bot }}$; or there is some arc that either has both endpoints on $\Gamma_{i}^{\mathrm{top}}$ or both endpoints on $\Gamma_{i}^{\mathrm{bot}}$. If there is a thick region of $\mathcal{T}_{i}{ }^{1}$ in $M_{i}$ then we are in a position to apply Lemma 17. Otherwise we are in a position to apply either Lemma 24 or Lemma 25.

We will describe the step that takes us from $\left(M_{i}, \Sigma_{i}, \Gamma_{i}\right)$ to $\left(M_{i+1}, \Sigma_{i+1}, \Gamma_{i+1}\right)$. First consider the initial step. If at the first step we encounter a thick region in $M_{0}$, then start with a leaf $L_{0}$ in a thick region of $F_{0}$ intersecting $\mathcal{T}^{2}$ in normal arcs and simple closed curves as is guaranteed by Claim 18. Applying Lemma 17 we obtain a collection $G_{0}$ of normal surfaces and at most one almost normal surface obtained by compressing $L_{0}$ to one side. If $G_{0}$ contains an almost normal surface and $L_{0}$ is incompressible above and below then $G_{0}=L_{0}$ is an almost normal surface isotopic to a leaf and we 
are done. If $G_{0}$ does not contain an almost normal surface isotopic to $L_{0}$ then without loss of generality let $G_{0}=\Gamma_{1}^{\text {top }}$ and proceed as below.

Henceforth assume without loss of generality that $L_{0}$ compresses above to give $G_{0}$. Otherwise we can invert the picture and declare $\Gamma_{i}^{\text {bot }}$ to be the "top" leaf. Since $G_{0}$ has been obtained by compressing above, Lemma 16 implies that $G_{0}$ is incompressible below. By Lemma 15 we can isotope the almost normal surface $G_{0}$ to be normal. This gives a new collection $\Gamma_{1}^{\text {top }}$ of normal surfaces isotopic to $G_{0}$. Cut $M_{0}$ along the collection $\Gamma_{1}^{\text {top }}$ and keep the component to the incompressible side below $\Gamma_{1}^{\text {top }}$ that contains part of $\partial M_{K}$. Call this submanifold $M_{1}$. Observe that $\Gamma_{1}^{\text {top }} \subset \partial M_{1}$. The cores of the tubes of the thick leaf that were compressed to give the almost normal surface $G_{0} \simeq \Gamma_{1}^{\text {top }}$ form the required 1-complex $\Sigma_{1}^{\text {top }}$. Let $\Sigma_{1}$ denote the pair $\Sigma_{1}^{\text {top }}$ and $\Sigma_{1}^{\text {bot }}=\Sigma_{0}^{\text {bot }}$ and let $\Gamma_{1}$ denote the pair $\Gamma_{1}^{\text {top }}$ and $\Gamma_{1}^{\text {bot }}=\Gamma_{0}^{\text {bot }}$. This completes the first step.

The remainder of the proof falls into the following three cases.

Case $1 M_{i}$ contains a thick region of $\mathcal{T}_{i}^{1}$ with respect to $F_{i}$.

In this case using Claim 18 start with a leaf $L_{i}$ in a thick region of the foliation $F_{i}$ intersecting $\mathcal{T}^{2}$ in normal arcs and simple closed curves disjoint from the 1-skeleton. Applying Lemma 17 we obtain a collection $G_{i}$ of normal surfaces and at most one almost normal surface obtained by compressing $L_{i}$ to one side. Lemma 16 implies that $G_{i}$ is incompressible to the opposite side. If $L_{i}$ is incompressible then $G_{i}=L_{i}$ and since $L_{i}$ is isotopic to a leaf we are done. So suppose without loss of generality that $L_{i}$ is compressible above to give $G_{i}$. The cores of the tubes of $L_{i}$ that are compressed above to give $G_{i}$ will make up the spine $\Sigma_{i+1}^{\text {top }}$. Let $\Sigma_{i+1}$ denote the pair $\Sigma_{i+1}^{\text {top }}$ and $\Sigma_{i+1}^{\text {bot }}=\Sigma_{i}^{\text {bot }}$. By Lemma 15 we can isotope the almost normal surface $G_{i}$ to give a new collection $\Gamma_{i+1}^{\text {top }}$ of normal surfaces. Cut $M_{i}$ along the collection $\Gamma_{i+1}^{\text {top }}$ and keep the component to the incompressible side below $\Gamma_{i+1}^{\text {top }}$ that contains part of $\partial M_{K}$. Call this new submanifold $M_{i+1}$. Let $\Gamma_{i+1}$ denote the pair $\Gamma_{i+1}^{\mathrm{top}}, \Gamma_{i+1}^{\mathrm{bot}}=\Gamma_{i}^{\mathrm{bot}}$.

If on the other hand $G_{i}$ is compressible below then the cores of the tubes of $L_{i}$ that are compressed below to give $G_{i}$ will make up the spine $\Sigma_{i+1}^{\text {bot }}$. By Lemma 15 we can isotope $G_{i}$ to be normal and call the new collection of normal surfaces $\Gamma_{i+1}^{\text {bot }}$. Let $\Gamma_{i+1}$ denote the pair $\Gamma_{i+1}^{\text {top }}=\Gamma_{i}^{\text {top }}$ and $\Gamma_{i+1}^{\text {bot }}$. Cut $M_{i}$ along the collection of normal surfaces $\Gamma_{i+1}^{\text {bot }}$ and keep the component to the incompressible side above $\Gamma_{i+1}^{\text {bot }}$. Call this new submanifolds $M_{i+1}$. This completes the recursive step in this case.

Case $2 M_{i}$ contains no thick region of $\mathcal{T}_{i}^{1}$ and some arc of $\mathcal{T}_{i}^{1}$ either has both endpoints on $\Gamma_{i}^{\text {top }}$ or has both endpoints on $\Gamma_{i}^{\text {bot }}$. 
Without loss of generality suppose that there is an $\operatorname{arc}$ of $\mathcal{T}_{i}^{1}$ with both endpoints on $\Gamma_{i}^{\text {top }}$. Applying Lemma 24, starting with a leaf $L_{i}$ of $F_{i}$ near the top singular leaf above all of the minima we obtain an almost normal surface $G_{i}$ in $M_{i}$ by compressing the leaf $L_{i}$ above. It follows from Lemma 16 that $G_{i}$ is incompressible below. Moreover, $\chi\left(G_{i}\right)=\chi\left(\Gamma_{i}^{\text {top }}\right)-2$. Using Lemma 15 isotope the almost normal surface $G_{i}$ to give a normal surface $\Gamma_{i+1}^{\text {top }}$. Cut $M_{i}$ along $\Gamma_{i+1}^{\text {top }}$ and keep the component to the incompressible side below $\Gamma_{i+1}^{\text {top }}$ to obtain the submanifold $M_{i+1}$. Denote by $\Gamma_{i+1}$ the pair $\Gamma_{i+1}^{\mathrm{top}}$ and $\Gamma_{i+1}^{\mathrm{bot}}=\Gamma_{i}^{\mathrm{bot}}$. The spine $\Sigma_{i+1}^{\mathrm{top}}$ of $M_{i+1}$ consists of the cores of the tubes of $L_{i}$ that are compressed above to give $G_{i}$. Denote by $\Sigma_{i+1}$ the pair $\Sigma_{i+1}^{\text {top }}$ and $\Sigma_{i+1}^{\mathrm{bot}}=\Sigma_{i}^{\mathrm{bot}}$.

In both Cases 1 and 2 the new surface $G_{i}$ isotopic to $\Gamma_{i+1}^{\text {top }}$ (resp. $\Gamma_{i+1}^{\text {bot }}$ ) in $M_{i}$ is not parallel as a normal surface to the normal surfaces $\Gamma_{i}^{\text {top }}$ (resp. $\Gamma_{i}^{\text {bot }}$ ). The reason depends on whether Lemma 17 or Lemma 24 was applied. If the surface, without loss of generality say $\Gamma_{i+1}^{\text {top }}$, comes from compressing a thick leaf via Lemma 17 then there is a subarc of $\mathcal{T}^{1}$ lying between $\Gamma_{i}^{\mathrm{top}}$ and $\Gamma_{i+1}^{\mathrm{top}}$ with both ends on $\Gamma_{i+1}^{\mathrm{top}}$. Hence $\Gamma_{i}^{\mathrm{top}}$ and $\Gamma_{i+1}^{\text {top }}$ are not parallel. If $\Gamma_{i+1}^{\text {top }}$ comes via Lemma 24 then $\chi\left(\Gamma_{i+1}^{\text {top }}\right)=\chi\left(\Gamma_{i}^{\text {top }}\right)-2$ so the surfaces are not parallel. If $\Gamma_{i}^{\text {top }}$ and $\Gamma_{j}^{\text {top }}$ are parallel then all leaves $\Gamma_{k}^{\text {top }}$ where $i \leq k \leq j$ are parallel as well. In particular, then $\Gamma_{i+1}^{\text {top }}$ is parallel to $\Gamma_{i}^{\text {top }}$ which cannot happen as we have just seen above. Therefore it follows that $\Gamma_{i}^{\text {top }}$ is non-parallel to $\Gamma_{j}^{\mathrm{top}}$ for all $i<j$.

Case $3 M_{i}$ contains no thick region of $\mathcal{T}_{i}^{1}$ and each $\operatorname{arc}$ of $\mathcal{T}_{i}^{1}$ has one endpoint on $\Gamma_{i}^{\text {top }}$ and one endpoint on $\Gamma_{i}^{\text {bot }}$.

In this case by Lemma $25 M_{i}$ is a product. Suppose $i \neq 0$. The submanifold $\overline{M_{i}}=$ $M_{i} \cup N\left(K_{i}\right)$ is a product as well and has the surface $S$ as a Heegaard surface that gives an irreducible Heegaard splitting of $\overline{M_{i}}$. By [12] it follows that the splitting surface is isotopic to $\Gamma_{i}^{\text {top }}$ and $\Gamma_{i}^{\text {bot }}$, one of which is in turn isotopic to the almost normal surface $G_{i-1}$ and so we are done.

If $i=0$ then the argument above shows that the surface $S_{K}$ consists of a collection of annuli. However the surface $S_{K}$ is a bridge surface for $K$ so it is connected. Therefore $S_{K}$ consists of one annulus and $K$ must be the unknot.

It follows from a well known result of Haken that there are only a finite number of non-parallel, disjoint, normal surfaces in $M_{K}$. See Haken [7]. Therefore we will only have to apply Lemma 24 and Lemma 17 a finite number of times before we either reach a situation where we apply Lemma 25 and obtain an almost normal surface isotopic to 
the bridge surface $S_{K}$ or we exhaust all of the non-parallel, disjoint, normal surfaces in $M_{K}$ and we obtain an almost normal surface isotopic to $S_{K}$.

\section{References}

[1] D Bachman, Heegaard splittings with boundary and almost normal surfaces, Topology Appl. 116 (2001) 153-184 MR1855961

[2] D Bachman, Thin position with respect to a Heegaard surface, preprint

[3] A J Casson, C M Gordon, Reducing Heegaard splittings, Topology Appl. 27 (1987) 275-283 MR918537

[4] A Coward, Algorithmically detecting the bridge number of hyperbolic knots (2006) arXiv:0710.1262

[5] C Frohman, The topological uniqueness of triply periodic minimal surfaces in $\mathbf{R}^{3}$, J. Differential Geom. 31 (1990) 277-283 MR1030674

[6] D Gabai, Foliations and the topology of 3-manifolds. III, J. Differential Geom. 26 (1987) 479-536 MR910018

[7] W Haken, Theorie der Normalflächen, Acta Math. 105 (1961) 245-375 MR0141106

[8] W Jaco, D Letscher, H Rubinstein, One vertex, ideal, and efficient triangulations of 3-manifolds, in preparation

[9] W Jaco, J H Rubinstein, 0-efficient triangulations of 3-manifolds, J. Differential Geom. 65 (2003) 61-168 MR2057531

[10] S King, Almost normal Heegaard surfaces (2003) arXiv:math.GT/0303377

[11] JH Rubinstein, Polyhedral minimal surfaces, Heegaard splittings and decision problems for 3-dimensional manifolds, from: "Geometric topology (Athens, GA, 1993)", AMS/IP Stud. Adv. Math. 2, Amer. Math. Soc., Providence, RI (1997) 1-20 MR1470718

[12] M Scharlemann, A Thompson, Heegaard splittings of (surface) $\times I$ are standard, Math. Ann. 295 (1993) 549-564 MR1204837

[13] M Scharlemann, M Tomova, Uniqueness of bridge surfaces for 2-bridge knots, Math. Proc. Cambridge Philos. Soc. 144 (2008) 639-650 MR2418708

[14] M Stocking, Almost normal surfaces in 3-manifolds, Trans. Amer. Math. Soc. 352 (2000) 171-207 MR1491877

[15] A Thompson, Thin position and the recognition problem for $S^{3}$, Math. Res. Lett. 1 (1994) 613-630 MR1295555

[16] M Tomova, Multiple bridge surfaces restrict knot distance, Algebr. Geom. Topol. 7 (2007) 957-1006 MR2336246 
Department of Mathematics and Statistics, California State Polytechnic University Pomona, 3801 West Temple Ave, Pomona, CA 91768, USA robinwilson@csupomona.edu

Received: 6 October 2007 